\title{
Robotic Implantation of Intracerebral Electrodes for Deep Brain Stimulation
}

\author{
C. Faria ${ }^{1 *}$, E. Bicho ${ }^{1 *}$, M. Rito ${ }^{3}$, L. Louro ${ }^{1}$, S. Monteiro ${ }^{1}$ and W. Erlhagen ${ }^{2}$ \\ ${ }^{1}$ Department of Industrial Electronics and Center Algoritmi, University of Minho, Portugal \\ ${ }^{2}$ Department of Mathematics and Applications and Center of Mathematics, University of Minho, Portugal \\ ${ }^{3}$ Service of Neurosurgery, Coimbra University Hospital, Portugal \\ *cfaria@dei.uminho.pt; estela.bicho@dei.uminho.pt
}

\begin{abstract}
This dissertation objective is to contribute for the development of a robotic system towards neurosurgery assistance in Deep Brain Stimulation (DBS) stereotactic procedures. Being DBS neurosurgery typically a long, physically and cognitively demanding procedure; the introduction of a robotic assistant to hold, manipulate and position instrumentation would improve the medical team working conditions and lead to better surgery outcomes. Upon understanding how could the robot be used and what robotic systems were adequate to the task, we implemented a simulation environment to emulate several industrial robot manipulators and the operating room. It was also developed each robot geometric and differential kinematic equations, and control algorithms specifically oriented for DBS neurosurgery assistance. Taking into account the operating room arrangement, the robot characteristics and task requirements, we selected the most apt industrial robotic manipulator and further elaborated on its placement and orientation to achieve utmost performance.
\end{abstract}

Index Terms-Robotic neurosurgery; Stereotactic electrode placement; Deep Brain Stimulation (DBS)

\section{INTRODUCTION}

Deep Brain Stimulation is a technique used in functional neurosurgery to stimulate specific basal ganglia regions via implanted electrodes. These electrodes are connected to a neuropacemaker that generates precise and controlled electrical signals [1]. DBS treatment (with different stimulation parameters) relieves symptoms of neurological disorders, ranging from: Parkinson's disease, epilepsy, dystonia, essential tremor and even psychiatric related pathologies. Recent epidemiology studies show the tremendous and growing impact of each of these pathologies in today's society [7] [9] [13].

The DBS treatment has earned a strong reputation among the neurologist community, due to the resulting symptomatic relief achieved, through a reversible procedure with few known side-effects. On the other side, the standard DBS surgery course of action meets several repetitive, iterative and timeconsuming - yet precision demanding steps [11] [14]. Thus comes the main motivation for our dissertation work, which

\section{Master Thesis}

Portuguese chapter of IEEE EMBS

$3^{\text {rd }}$ Portuguese Meeting in Bioengineering, February 2013

University of Minho involves the development of a robotic system to perform these repetitive and menial tasks in collaboration with the medical team. The projected neurosurgery robot, or as we like to call it, an 'intelligent surgical tool' should therefore: place, hold or precisely manipulate other surgical instrumentation and also integrate safety check routines.

Robotic technology continuous progress, have brought its precision and repeatability standards to a limit that far exceeds human's capabilities [3]. However, artificial intelligence can not compete with neurosurgeon's dexterity, judgement experience among other advantages [2]. Our aim is to bring forth the best of both worlds, in other to improve the medical team working experience and potentially improve the surgery outcome, through precise and consistent methodologies. Furthermore, we found that the price of an entire robotic system is roughly a limiting factor, since it is about half the cost of a simple mechanical not-actuated stereotactic device used in DBS surgery simply to position electrodes.

Facing the extremely challenging task of developing an entire robotic system oriented to surgery in the context of a master thesis, we set as objective: to contribute for the development of a robotic system towards neurosurgeon's assistance in DBS procedures. We took the first steps towards understanding: what should the robot do in a DBS neurosurgery; what is already done and what can be improved in neurosurgery robots; and developing an initial virtual robotic solution not only to test several robot systems but also to address anticipated real implementation issues.

In section II it will be presented the course of action of a standard DBS neurosurgery and will be emphasized how should the robot be of use; in section III we will summarily present the state of the art in robotic neurosurgery for stereotactic procedures. In section IV, we will present the choice process of the industrial robotic systems along with the developed kinematic equations for each; and in section $\mathrm{V}$ the virtual tool created to test the robotic systems and the implemented control algorithms. To conclude in section VI, it will be presented the first results and in section VII we will describe the conclusions of this dissertation, and point out future work. 


\section{Deep BRain Stimulation}

The first task of the dissertation was to understand the robot utility in a DBS neurosurgery. To do so, we started by attending to a standard DBS neurosurgery that took place in Coimbra University Hospitals (Portugal), and was conducted in a Parkinson's disease affected patient.

\section{A. Neurosurgery Procedure}

After being selected for DBS neurosurgery, the patient undergoes imaging scans to pinpoint the brain structures to be stimulated, as well as the skull entry coordinates through which, each electrode will pass to reach the stimulation coordinates. Both coordinates are extrapolated based in a stereotactic reference frame ${ }^{1}$ (fig. 1a) that is previously fixed to the patient's skull. Along the patient's preparation, the coordinates of the stimulation target are simulated via a phantom device (fig. 1b). The mechanical screws of the stereotactic frame mounted in the phantom, are set to position a driver system that will guide the electrodes past the skull entry point towards the stimulation target.

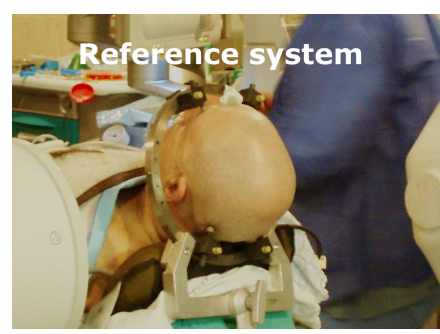

(a) Stereotactic Reference System.

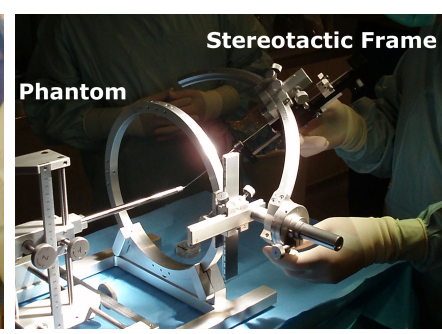

(b) Surgical coordinates verification.
Fig. 1. Intraoperative surgery preparation steps.

Once verified the phantom and frame coordinates for one stimulation target, the stereotactic frame is dismounted from the phantom and attached to the reference system on the patient's head (fig. 1a). The stereotactic frame is once again set to mark the skull entry point, and then moved aside to clear the neurosurgeons' workspace. With the skull entry point, the neurosurgeon team proceeds to incise the scalp and drill a burr hole in the patient's skull.

The stereotactic frame is positioned one final time to assist the neurosurgeons descend several sets of electrodes towards the stimulation target (to register brain activity or to apply stimulation signals). Once the electrodes are placed, the stimulation variables like: electrode depth and signal intensity are adjusted, according to the variation of the patient's symptoms ${ }^{2}$.

If the DBS surgery is bilateral, all the procedure must be thoroughly repeated for another stimulation target.

\section{B. Robotic System Utility}

Based on the information gathered about DBS surgery, we thought of several potential improvements to the standard surgery with the introduction of a robotic system:

\footnotetext{
${ }^{1}$ In this case, it was a ring-shaped structure.

${ }^{2}$ The patient sedation is lowered and the patient remains awake during the symptoms evaluation by neurophysiologists.
}

1) Interface the imaging software - where the surgery coordinates are generated - with the robotic controller;

2) Avoid mount/dismount and the process of setting the stereotactic frame coordinates, by making the procedure 'frameless';

3) Assist the neurosurgeon in skull drilling, by constraining the trepan trajectory;

4) Swiftly position and manipulate instrumentation with guaranteed precision and consistency;

5) Assist highly experienced senior neurosurgeons that might lack the required dexterity; or guide young and much less experienced neurosurgeons.

\section{StATE OF THE ART}

Having outlined the robotic system goal, it was essential to perform a state of the art search on current neurosurgical robot solutions oriented to stereotactic brain surgeries. We searched on MeRoDa (medical robotics database) and in several review papers, for robotic systems that fall in the above cited category and found some oriented and potentially adaptable systems [5] [12] [6].

Among the robotic systems oriented to minimally invasive stereotactic neurosurgery we selected: i) Neurobot, developed in Imperial College of Science, Technology and Medicine in London; ii) NeuroMate, by Renishaw; iii) Pathfinder, by Prosurgics Ltd.; iv) Robocast, from Neuroengineering and medical robotics laboratory of Politecnico di Milano and v) Rosa, by Medtech. The Neurobot and the Robocast robotic systems are still in development stage.

We also found neurosurgical robotic systems built towards other surgical procedures (like endoscopic handling, instrumentation guidance and tele-operated actuation) that had the potential to be used in a stereotactic surgery like DBS. Among those we selected: Evolution1, by Universal Robot Systems, Minerva from the Microengineering laboratory in the Swiss Federal Institute of Technology and NeuroArm from the University of Carlgary. However, only the Evolution 1 system is currently available at market and according to the information we have, the Minerva project has been discontinued.

Upon analyzing each oriented and adapted system features and based on the information gathered about DBS surgery, it was possible to outline a set of desired and unwanted characteristics for our project. However, all state of the art systems presented one or another uninviting feature like:

1) Parallel manipulator arquitecture;

2) Rely on stereotactic frame;

3) Fixed robotic base, compromised mobility;

4) Need of an integrated imaging machine (MRI/CT);

5) Have integrated imaging/planning softwares;

6) Complex system architectures;

7) Exaggerated costs.

We seek a serial actuator with 6 or more degrees of freedom (DOF), for increased flexibility and broader workspace with the ability to reach specific positions with no orientation restriction. The robot should have stiff and lethargic movements to avoid further stress/overheat in its joints and also to facilitate 
motion stop action since the movement inertia is smaller. However, the choice of the robot is primarily conditioned by precision and repeatability since the slightest deviation from a target deep brain structure can reproduce very different stimulation effects (reference).

In term of robotic system features, we concluded that it should optimally include: i) a mobile platform, to be easily moved inside or outside the operating room; ii) vision system, so the robot can recognize its position relative to the surgical referential and also to have feedback on the surrounding environment (avoid collisions); iii) have an integrated or 'an interface with' the imaging/planning software - where neurosurgeons extrapolate surgical coordinates - used by the healthcare institution; iv) Have simple, intuitive usage and keep low acquisition and maintenance costs.

\section{INDUSTRIAL Robot SELECTION}

Having defined a the sought robotic system characteristics, we searched the most renowned industrial robotic producers, for robotic systems that fit in the outlined profile. We selected 25 serial manipulator systems from companies like: $A B B$, Adept, Epson, Fanuc, Kuka, Mitsubishi, Motoman, Nachi, Schunk, Stäubli, Toshiba and Universal Robots.

Starting from 25 systems, we conducted several comparative analyses with the parameters displayed at the producers data sheets, such as controller and robot weight, horizontal reach, robot repeatability and payload capacity. We assigned a limit threshold to each variable and in the end we reduced our potential robotic system pool from 25 to 3 robots from:

- $A B B$, a 6 DOF serial manipulator;

- Motoman, a 6 DOF serial manipulator;

- Schunk, a 7 DOF serial manipulator (also to assess the impact of the extra degree of freedom).

Other specifications like the manipulator dimensions, structure and joint limits could not be directly compared between systems, since its implication depends on the robot workspace and in the task characteristics. To test each of this variables we implemented a virtual instance of each robot in a simulator (cf. section $\mathrm{V}$ ).

Robotic tasks can be divided into small sequences of pointto-point or velocity/acceleration specified motions. The robot manipulator movement is executed through the control of each joint along its kinematic chain. Therefore it is essential to map the relationship between the space coordinates where the robot operates (Cartesian space) to the robot joints' positions (Joint space).

The problem of Direct Kinematics, expresses the forward relation between the robot joint values and the resulting cartesian coordinates. On the other hand, the problem of Inverse Kinematics expresses the set of joint values to achieve a desired/input position, velocity or force in the cartesian space. For the 3 selected manipulators, we developed the Geometric and Differential Kinematics for the Direct and Inverse problems. Geometric Kinematics allowed us to control the robot based on cartesian and joint positions and were one essential tool for positioning and holding surgery tasks. Differential Kinematics consider velocity and acceleration/force values instead, and were used in the manipulation of surgical instrumentation based in velocities and relative positions (straight line motion).

Detailed information about the Kinematics developed can be found at [4].

\section{IMPLEMENTED SOLUTION}

At this point, we had developed a low level control algorithm based in desired positions and velocities for the 3 candidate robotic systems. Further assessment of each manipulator should be carried either using the real robot or through a simulation environment. Since robotic manipulators are not a cheap and of easy access tool, we resorted to a robotic simulator to test the kinematic equations and to implement the control strategies oriented to the surgery tasks.

\section{A. Robotics Simulator}

The simulator should emulate the selected robots' features and dimensions, and also the operating room environment. To understand how to better fit the robot in its workspace and how to adapt the control approach to the surrounding elements, it is essential to recreate the operating room. Thus, we sought a open source software to implement each of the specific elements, either robots and virtual world. In addition to the custom components, the simulator should include both graphical and physical representations of each virtual component.

According to the aimed features, considering the available alternatives and the local shared knowledge, we chose to develop our solution in a $3 \mathrm{D}$ robotics simulator created in-house, the CoopDynSim. Besides gathering all the cited features, it also facilitates the code portability between simulation and real robot, due to the abstraction of the communication layer between the control application and the robot either virtual or real [10].

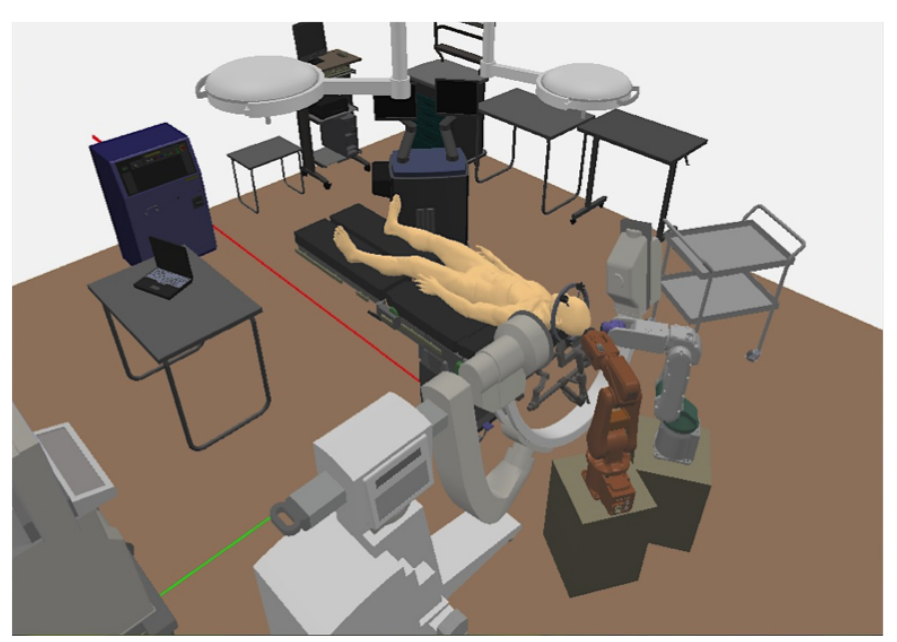

Fig. 2. CoopDynSim, operating room emulated environment.

CoopDynSim is built on $\mathrm{C}++$, uses OpenGL graphical library and runs on NewtonGD physics engine. It was originally 
developed for mobile robots and basic shaped environments. Thus, one of our tasks involved importing and designing 3D models of medical equipment from the Neurosurgery Service of Coimbra University Hospitals operating room (fig. 2).

The 3 selected industrial robots were also included in the simulator. The $3 \mathrm{D}$ models of each robot were provided at the producers website. We did slight changes to the 3D models in order to remove superfluous details and parts and thus reduce the computational cost of drawing these models. We added a physical representation to each link of each robot, who used by the physics engine to emulate the mass, form, and physical interactions of each part.

At this point the robots were just a set of scattered links, which we had to connect using hinge physical joints according to the robot architecture. It was implemented virtual actuators with PID controllers that respond to desired joint position and velocity inputs.

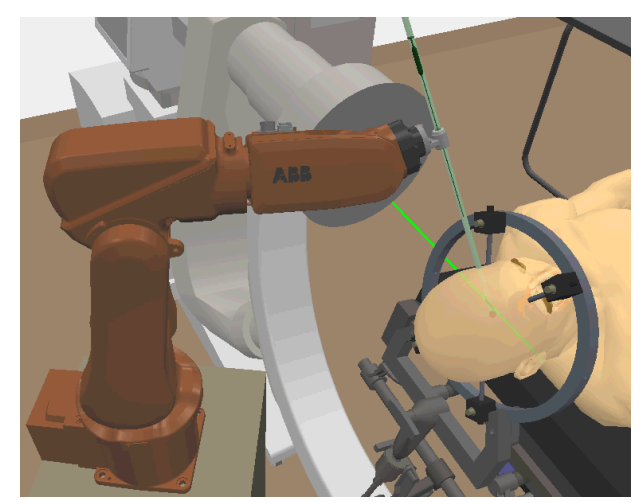

Fig. 3. $A B B$ robot following a DBS surgery pre-defined trajectory.

We implemented specific DBS surgery end-effectors, including the trepan tool and electrode holding device, both with a linear actuator, to be moved independent from the manipulator. Furthermore, we created a feature called Surgical Plan, which allows the simulator user to visualize the surgery targets and trajectories in the virtual world (represented by physicless, semi-transparent marks). This functionality facilitated visualization of target and trajectories entities and aided the debug process of the control application, fig. 3 .

\section{B. Control Application}

With the virtual operating room and robots, we started developing the control application. Given the abstraction provided by the communication layer, we chose to implement the control application in MatLab due to the quick, ease algorithm testing and also to fulfill a request of the medical team we are cooperating with, since it is a programming language known from part to part.

The control application was built in several modules/classes: i) Communication, ii) Robots, iii) DBS, iv) Kinematics and v) Utilities, all centered/connected to User-Interface (UI), fig. 4.

The control application establishes the communication between the server ports associated to the robot modules, and the client ports linked to the control application. In the Developer

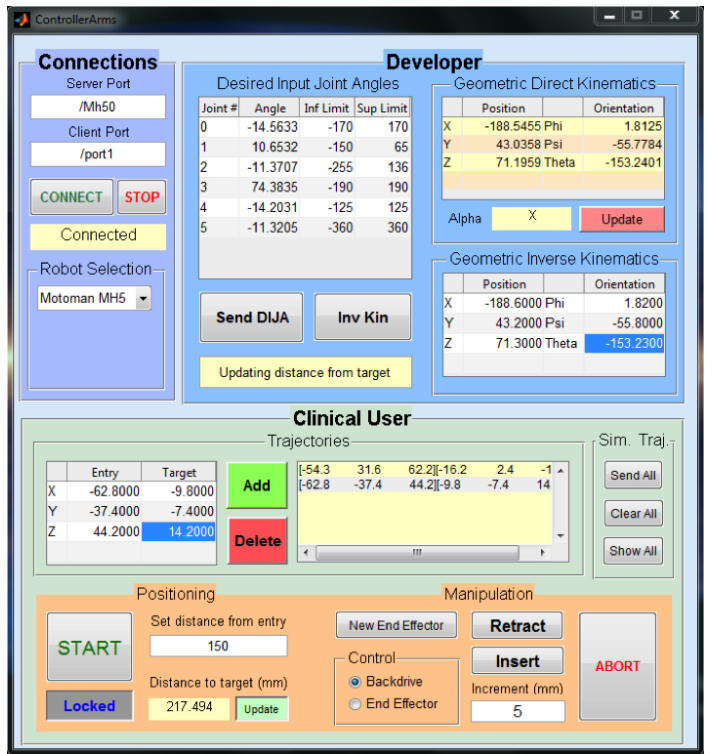

Fig. 4. Control Application User-Interface.

panel, the application lets the user control individual robot joints, control the robot by inputing a desired position and orientation and read, in real time, the robot end-effector position relative to a surgery defined referential.

The Clinical User panel has a feature that allows the user to manage the surgery coordinates to be followed by the robot. Most of the actions in DBS surgery are performed along a straight line, from the skull entry point towards the target to be stimulated. Thus, the user is asked to introduce these two sets of $3 \mathrm{D}$ coordinates for each target to be stimulated. The control application communicates with the simulator world, which creates the trajectory and the target marks relative to the surgery referential. The clinical user can insert, remove one or several trajectories/targets.

When the user selects one trajectory/target to be stimulated, if there are other marks added to the Surgical Plan, they will be hidden so the user knows exactly where the robot will actuate. From here we split the control approach algorithm in two parts:

1) Positioning - where the robot will make the initial approach to the linear trajectory of electrode insertion;

2) Manipulation - the robot will be locked to this linear trajectory and will only execute upward or downward movements.

After selecting the desired trajectory, in the Positioning stage, the user should input the distance from the entry point and along the trajectory, where the robot end-effector should position. We also implemented a function to compute and show in real time the distance from the robot end-effector to the stimulation target.

At the Manipulation stage, the robot can only move along the locked trajectory. The control application starts by identifying what end-effector is attached to the hand of the manipulator (if any). The robot is expected to execute incremental 
movements toward or away from the target, that can either be accomplished by a joint action of the whole manipulator - resorting to Differential Kinematics - or by executing independent increments of the instrumentation (trepan and electrode drive) relative to the robot arm.

Safety is currently pointed as the most discouraging factor for the use of robotic instrumentation within operating rooms [8]. As such an important factor, we established safety routines in the control application. Firstly, motion restriction was created so the robot does not deviate from an assigned linear trajectory, thus preventing inadvertent movements. Additionally, when the trepan tool is attached to the manipulator hand, the robot stops any motion that would cause the drill to pass behind the skull entry point, to avoid brain damage. Precision control routines, compute the distance between the desired and the generated solution, position and orientation. If the generated position and orientation fall outside a safety threshold distance, the robot instead of moving, stops and notifies the user ${ }^{3}$. We also created a log file system, so the control application registers every user actions, variables and introduced values. Each $\log$ file is associated to a unique surgical procedure and it is registered the time (hh:mm:ss) when each event happened.

\section{REsults}

At this stage, we want to evaluate the performance of the developed system mainly in two areas: overall precision and task suitability/system flexibility. It was impossible to assess the precision of the selected robots and control approach, due to the unavailability of information regarding the robot joint controllers and manipulator precision.

However, we assessed each robot flexibility and suitability performance towards the required task, taking into account their structure and possible conflicts with the surrounding operating room environment. To do so, we developed a simple task where we tested several combinations of: robot type; robot base platform heights, relative to the patient's head; robot orientation, relative to the patient's sagittal plane; for 14 generic trajectories depict in, fig. 5.

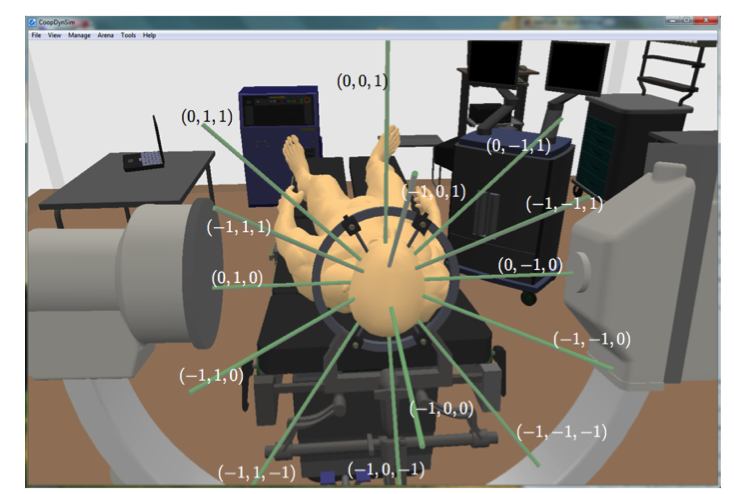

Fig. 5. Selected robots flexibility test.

\footnotetext{
${ }^{3}$ These safety checks are performed before any action (Positioning and Manipulation stages).
}

Each cell of $6 a, 6 b$ or $6 c$ represents a set of 14 trajectories for a specific robot height (of robot's end-effector relative to the patient's head height, in home position) and orientation (of robot's home position posture relative to the patient's sagittal plane $-0^{\circ}$ when the robot is in front of the patient).

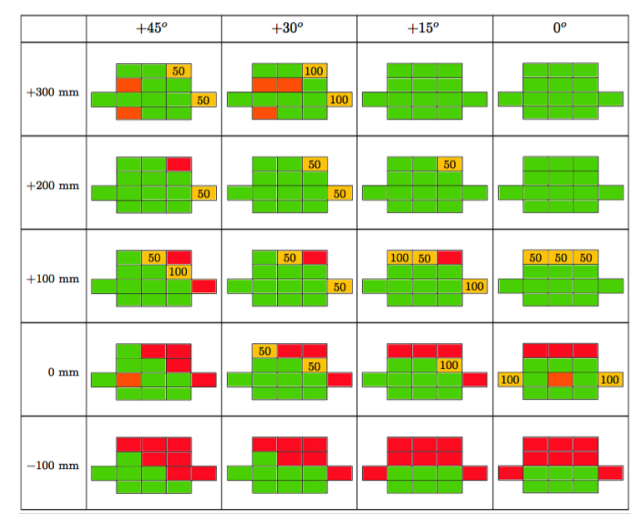

(a) ABB robot flexibility results.

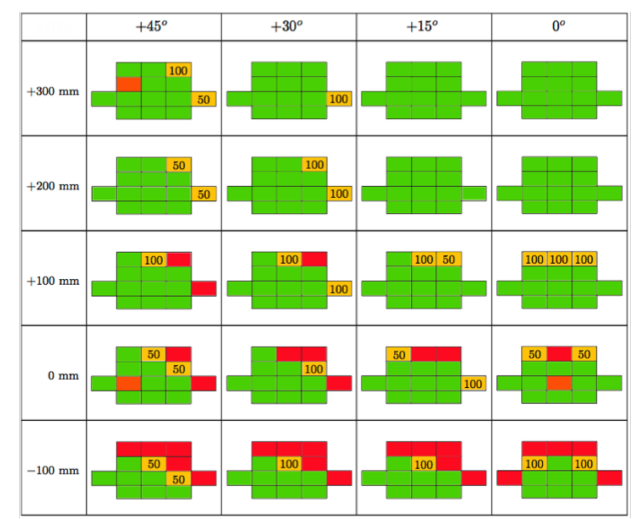

(b) Motoman robot flexibility results.

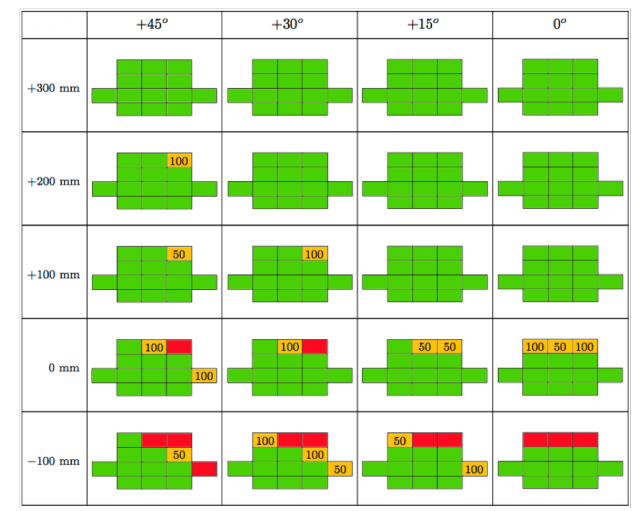

(c) Schunk robot flexibility results.

Fig. 6. Flexibility test results for several robot base heights and robot orientation relative to the patient. Green, robot can reach $150 \mathrm{~mm}$ from the skull entry point along the insertion trajectory and move towards the target. Yellow, robot can reach $\mathrm{Xmm}$ from the skull entry point along the insertion trajectory and move towards the target. Orange, robot can reach the insertion trajectory but collides with end-effector instrumentation. Red, robot can not reach the insertion trajectory.

Comparing the results of both 6 DOF manipulators $(A B B$ robot and Motoman robot) we see that for starting end-effector 
heights equal or below the patient's head the robot fails to reach several trajectories, even the most probable ones (6 central trajectories). For the remaining heights $(+100 \mathrm{~mm}$, $+200 \mathrm{~mm}$ and $+300 \mathrm{~mm}$ ), both robots can reach most of the defined trajectories, and with success the most probable ones. However, we suggest as preferable starting end-effector's height $+200 \mathrm{~mm}$, because for $+100 \mathrm{~mm}$ the trajectories on the opposite side of the robot manipulator relative to the patient's sagittal plane are reached at the limit of the robot's workspace, which can comprise its stability and precision. At $+300 \mathrm{~mm}$ height, the robot collides with the end-effector instrumentation for the most central trajectories for greater orientation angles.

We point as preferable robot orientation angle between $+15^{\circ}$ and $+30^{\circ}$, since the robot achieves better results for smaller angles. At the same time, the robot occupies more of the neurosurgeon's workspace for smaller orientation angles.

On the other hand, the 7 DOF Schunk robot presents the best flexility results, followed by Motoman robot with $A B B$ robot, which shows the worst flexibility among the selected systems. The extra DOF and the larger arm horizontal reach, enable the Schunk robot to successfully reach most of the trajectories for almost every combination of robot base height and orientation. Thus, if workspace conditions are a problem, a 7 DOF robotic arm is a option to consider.

However, the 7 DOF manipulator, unlike the others, collided several times with the surrounding equipment, mainly due to the elbow redundancy caused by the extra $\mathrm{DOF}^{4}$. Furthermore, the extra joint in a serial manipulator will add another error source to the end-effector final position and orientation. Hence the need to further invest in joint actuators precision, which will ultimately enhance the final product cost.

For these reasons and facing the results achieved so far, we dare to point Motoman robot as the most fit robotic system for DBS surgery assistance.

\section{CONCLUSIONS}

The knowledge acquired about DBS surgery, the close contact with field professionals - which may later turn to endusers of this project - and by witnessing what was already achieved with robotic systems in stereotactic neurosurgery, allowed us to outline a set of desirable features for the sought robotic system. We compiled information about the existing mass produced industrial robots, to select the most suited for our purpose. Based on the selected systems, we developed specific control algorithms oriented to DBS surgery tasks, and created several tools to test and consolidate the control application. With these tools, it was possible to assess each manipulators' flexibility, suitability to the assigned tasks, and to analyze their interaction with the operating room medical equipment.

In terms of future work, it includes devising a mobile platform so the robot can be easily moved inside and outside the operating room and an attach system to fix the mobile

\footnotetext{
${ }^{4}$ The control application cost functions, still don't include collision avoidance routines. Nonetheless, it was evident the disparity between the number of collisions occurring between 7 and 6 DOF manipulators.
}

platform to the patient's reference stereotactic system. Another goal to achieve is a system to recognize the robot's position and orientation relative to the surgical referential, as this transformation isn't static.

Most importantly, the information gathered so far reinforced our perspectives about the viability of this new and promising project, due to the availability of the resources needed to achieve a final solution and to the fact that it gathers a set of attractive features, not yet explored in the current market offer.

\section{ACKNOWLEDGEMENTS}

This work has been partially financed by projects FP7 Marie Curie ITN - NETT (project $\mathrm{n}^{\circ} 289146$ ), FCT FCOMP-01-0124-FEDER-022674, Pest-C/MAT-UI0013/2011 (FCT grant ref. UMINHO/BIC/8/2012) and FCT PhD grant (ref. SFRH/BD/86499/2012).

\section{REFERENCES}

[1] A. L. Benabid. Deep brain stimulation for Parkinson's disease. Current Opinion in Neurobiology, 13(6):696-706, 2003.

[2] D. B. Camarillo, T. M. Krummel, and J. K. Salisbury. Robotic technology in surgery: past, present, and future. American journal of surgery, 188(4A Suppl):2S-15S, October 2004.

[3] F. Cardinale and R. Mai. Robotic Implantation of Intracerebral Electrodes in Epilepsy Surgery. Congress of Neurosurgical Surgeons Spring, 12(2):24-26, 2011.

[4] C. Faria. Robotic Implantation of Intracerebral Electrodes for Deep Brain Stimulation. Master thesis, University of Minho, 2012.

[5] T. Haidegger, L. Kovacs, G. Fordos, Z. Benyo, P. Kazanzides, A. Katashev, Y. Dekhtyar, J. Spigulis, and R. Magjarevic. Future Trends in Robotic Neurosurgery. In Alexei Katashev, Yuri Dekhtyar, and Janis Spigulis, editors, 14th Nordic-Baltic Conference on Biomedical Engineering and Medical Physics, volume 20 of IFMBE Proceedings, pages 229-233, Riga, 2008. Springer Berlin Heidelberg.

[6] C. S. Karas and E. A. Chiocca. Neurosurgical robotics: a review of brain and spine applications. Journal of Robotic Surgery, 1(1):39-43, 2007.

[7] L. Lau and M. Breteler. Epidemiology: Parkinson disease. The Lancet Neurology, 5:525-35, 2006.

[8] S. Lavallee, J. Troccaz, L. Gaborit, P. Cinquin, A. L. Benabid, and D. Hoffmann. Image guided operating robot: a clinical application in stereotactic neurosurgery. In Proceedings 1992 IEEE International Conference on Robotics and Automation, pages 618-624, Nice, 1992. IEEE Comput. Soc. Press.

[9] E. D. Louis and R. Ottman. How common is the most common adult movement disorder? Estimates of the prevalence of essential tremor throughout the world. Movement disorders, 13(1):5-10, 1998.

[10] T. Machado, M. Sousa, S. Monteiro, and E. Bicho. CoopDynSim: a 3D robotics simulator. In Robotica, pages 45-50, Braga, 2012. University of Minho.

[11] Erwin B Montgomery Jr. and John T Gale. Mechanisms of action of deep brain stimulation (DBS). Neuroscience Biobehavioral Reviews, 32(3):388-407, 2008.

[12] N. Nathoo, M. C. Cavuolu, M. A. Vogelbaum, and G. H. Barnett. In touch with robotics: neurosurgery for the future. Neurosurgery, 56(3):421-33; discussion 421-33, March 2005

[13] Aidan Neligan and J. W. Sander. The incidence and prevalence of epilepsy. In Epilepsy 2011, volume 76, chapter 1, pages 1-7. International League Against Epilepsy's 13th, London, 2011.

[14] J. S. Perlmutter and J. W. Mink. Deep brain stimulation. Annual review of neuroscience, 29(1):229-57, January 2006. 